\title{
NF-кB signaling modulates radiation-induced microglial activation
}

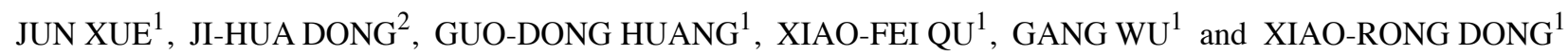 \\ ${ }^{1}$ Cancer Center, Union Hospital, Tongji Medical College, Huazhong University of Science and Technology; ${ }^{2}$ Union Hospital, \\ Tongji Medical College, Huazhong University of Science and Technology, Wuhan, Hubei 430022, P.R. China
}

Received January 21, 2014; Accepted March 18, 2014

DOI: $10.3892 /$ or.2014.3144

\begin{abstract}
Microglial activation has been suggested to be associated with the incidence of radiation-induced brain injury. The present study investigated the molecular mechanism(s) involved in radiation-induced activation of the microglia. Mouse microglial BV-2 cells were exposed to different doses of radiation. The release of inflammatory factors was evaluated by enzyme-linked immunosorbent assay and real-time reverse transcriptase polymerase chain reaction. Protein expression was determined by immunocytochemistry and immunoblotting. Microglial activation was induced by radiation [ $>16$ Gray (Gy)]. Activated cells exhibited a stouter spherical morphology and the levels of ionized calcium-binding adapter molecule-1 and CD68 were considerably upregulated. The generation of inflammatory factors, including interleukin-1 $\beta$ (IL-1 $\beta$ ), tumor necrosis factor- $\alpha$ (TNF- $\alpha$ ), IL-6, toll-like receptor 8 (TLR-8) and cyclooxygenase 2 (COX-2), was increased and peaked at either 3 or $6 \mathrm{~h}$ after radiation treatment. Phosphorylated $\gamma$-histone 2A, member X $(\gamma$-H2AX), which facilitates DNA double-strand breaks (DSBs), was upregulated at $3 \mathrm{~h}$ postradiation treatment. This was accompanied by the nuclear translocation of the nuclear factor $-\kappa \mathrm{B}(\mathrm{NF}-\kappa \mathrm{B})$ p 65 subunit. Moreover, $3 \mathrm{~h}$ following radiation treatment, the $\mathrm{NF}-\kappa \mathrm{B}$ essential modulator (NEMO) was markedly elevated, whereas the NF- $\kappa \mathrm{B}$ regulatory inhibitor- $\alpha(\mathrm{I} \kappa \mathrm{B}-\alpha)$ was considerably decreased. Our results demonstrate that the NF- $\kappa \mathrm{B}$ signaling pathway may trigger microglial activation and release of inflammatory factors following irradiation. These findings may provide valuable insight into understanding the molecular mechanism(s) involved in brain injury induced by radiation therapy.
\end{abstract}

Correspondence to: Dr Xiao-Rong Dong, Cancer Center, Union Hospital, Tongji Medical College, Huazhong University of Science and Technology, Wuhan, Hubei 430022, P.R. China

E-mail: xuejingling@whu.edu.cn

Key words: microglial activation, irradiation, NF- $\mathrm{KB}$ signaling pathway

\section{Introduction}

Cranial radiation therapy (CRT) is one of the most effective treatment modalities of primary and secondary brain tumors. However, the risk of CRT-induced injury to normal surrounding brain tissues is also associated with adverse side-effects. As many as $50 \%$ of CRT-treated brain cancer survivors develop late-onset radiation-induced injury of normal brain tissues, and pediatric cases account for a large portion of this population. The recent development and application of 3D-conformal and intensity-modulated radiation therapies, both of which deliver radiation more precisely to the tumor, have helped to reduce the amount of radiation-induced injury in surrounding normal tissues. Nevertheless, radiation-induced brain injury (RIBI) remains a common and severe side-effect of CRT. Survivors of irradiated childhood brain tumors who develop late-delayed cognitive deficits due to RIBI may present with learning disabilities as well as growth and psychomotor retardation.

Although the precise pathogenic mechanisms involved in the development and progression of RIBI have not yet been fully elucidated, a variety of cell types within the brain, including the microglia, astrocytes, oligodendrocytes, neurons and endothelial cells, have been determined to contribute to this process (1). The microglia, in particular, represent $\sim 10 \%$ of the total glial population in the central nervous system (CNS), and are the macrophage equivalent of the CNS where they serve as key mediators of neuroinflammation (2). Accumulating evidence suggests that activated microglia may contribute to RIBI (3). Chiang et al reported that 20-45 Gray (Gy) radiation could increase glial fibrillary acidic protein (GFAP) expression and induce astrocytic and microglial responses. These responses were associated with reactive gliosis and inflammation (4). In addition, conditioned medium collected from irradiated microglial cells has been shown to induce astrogliosis, which may contribute to radiation-induced edema (5).

Under normal physiological conditions, microglial cells react to a variety of stimuli, including lipopolysaccharide and interferon- $\alpha$. In vitro studies have shown that activated microglia produce a variety of pro-inflammatory mediators and cytokines, such as interleukin (IL)-1, reactive oxygen species, nitric oxide (NO) and prostaglandin E, all of which are thought to be responsible for inflammation-related diseases such as RIBI, trauma, ischemia, Alzheimer's disease and 
neural death. Similarly, in vivo studies have shown that brain irradiation leads to a marked increase in microglia activation and release of pro-inflammatory cytokines associated with inhibition of neurogenesis in hippocampus (6-9). Although the acute mechanism of microglial activation during RIBI remains unclear, it has been suggested that microglial activation is closely correlated with inflammation. For example, irradiating microglia leads to their activation and a marked elevation in the expression of proinflammatory genes, including tumor necrosis factor- $\alpha$ (TNF- $\alpha)$, IL-1 $\beta$, IL-6 and cyclooxygenase- 2 (COX-2) $(5,10,11)$.

DNA double-strand breaks (DSBs) are the most deleterious form of DNA damage and numerous in vitro studies have sought to elucidate the mechanism of the DSB repair system that is activated upon exposure to ionizing radiation. It has been determined that the presence of DNA DSBs can rapidly trigger activation of nuclear factor $(\mathrm{NF})-\kappa \mathrm{B}$ signaling pathway via the NF- $\kappa \mathrm{B}$ essential modulator (NEMO) $(5,6)$. The death-domain protein PIDD, which was originally identified as an early p53-inducible gene and is implicated in p53-induced apoptosis (5), also acts as a mediator of the DNA-damage-activated stress response and is involved in genotoxic stress-induced $\mathrm{NF}-\kappa \mathrm{B}$ activation $(6,7)$. PIDD expression enhances genotoxic-stress-induced NF- $\kappa \mathrm{B}$ activation through augmented sumoylation and ubiquitination of NEMO. Moreover, the NF- $\kappa \mathrm{B}$ signaling pathway and DNA DSBs have been linked to microglial activation induced by various stimuli, such as the inflammagen lipopolysaccharide $(12,13)$. Nevertheless, the involvement of NF- $\mathrm{B}$ and DNA damage in radiation-induced microglial activation remains to be clarified.

In the present study, we investigated the release of inflammatory factors, activation of NF- $\kappa \mathrm{B}$ and DNA DSBs in microglia BV-2 cells following irradiation. Our findings provide insight into the mechanism of microglial activationmediated brain injury during radiation therapy.

\section{Materials and methods}

Reagents. RPMI-1640 culture medium and fetal bovine serum (FBS) were purchased from Gibco (Grand Island, NY, USA). TRIzol reagent was obtained from Invitrogen (Carlsbad, CA, USA). The anti-Iba-1 rabbit antibody was purchased from Wako Chemical (Osaka, Japan) and the NEMO and I $\kappa$ B- $\alpha$ primary antibodies were purchased from Santa Cruz Biotechnology (Santa Cruz, CA, USA). The anti- $\gamma$-histone $2 \mathrm{~A}$, member $\mathrm{X}(\gamma-\mathrm{H} 2 \mathrm{AX})$ mouse monoclonal antibody was purchased from Abcam (Cambridge, MA, USA). The AlexaFluor 488 conjugated goat anti-mouse, donkey anti-goat secondary antibody and the AlexaFluor 568 conjugated goat anti-rabbit secondary antibody were purchased from Invitrogen. Vectashield mounting medium with 4',6-diamidino-2-phenylindole (DAPI) was purchased from Vector Laboratories (Burlingame, CA, USA). Mouse IL-1 $\beta$, TNF- $\alpha$ and IL-6 Quantikine enzyme-linked immunosorbent assay (ELISA) kits were purchased from R\&D Systems (Minneapolis, MN, USA). The Griess reagent NO assay kit was from Beyotime Biotech (Jiangsu, China). M-MLV reverse transcriptase and SYBR-Green I were purchased from Toyobo Company (Osaka, Japan). All primers and oligo(dT) were synthesized by Shanghai Invitrogen (Shanghai, China). The dNTP mix and Taq DNA polymerase were obtained from Fermentas International (Burlington, ON, Canada). Nuclear and cytoplasmic protein extraction kits were purchased from Bio-Rad Laboratories (Hercules, CA, USA). The enhanced chemiluminescence western blotting detection system was obtained from Millipore (Bedford, MA, USA). The p65 rabbit polyclonal antibody was purchased from Santa Cruz Biotechnology and the antibody against $\beta$-actin was from Sigma (St. Louis, MO, USA).

Cell culture. The mouse microglial cell line BV-2 was maintained in the Laboratory Center of Union Hospital, Tongji Medical College, Huazhong University of Science and Technology. Cells were cultured in RPMI-1640 culture medium supplemented with $10 \% \mathrm{FBS}$ at $37^{\circ} \mathrm{C}$ in a $5 \%$ $\mathrm{CO}_{2}$-humidified incubator. Cells in the logarithmic phase were used in the experiments.

Irradiation procedure. A cell suspension was prepared at a density of $2 \times 10^{5}$ cells $/ \mathrm{ml}$. A total of $10 \mathrm{ml}$ of the cell suspension was added to a 6 -well plate, which was placed $100 \mathrm{~cm}$ away from the radioactive source. Irradiation of the cells was performed with a ${ }^{137} \mathrm{Cs}$ irradiator (Siemens, Munich, Germany) at a dose rate of $2.0 \mathrm{~Gy} / \mathrm{min}$. After a single dose of radiation, cells were returned to the $5 \% \mathrm{CO}_{2}$ incubator. Control cells did not receive radiation treatment.

Immunocytochemistry. At the indicated time points following irradiation, cells were fixed, permeabilized and blocked with goat serum. Cell samples were stained with anti-Iba-1 (1:200 dilution), anti-CD68 (1:800), anti-NEMO (1:200) or anti-IкB- $\alpha(1: 200)$ primary antibodies and then probed with AlexaFluor 568 or AlexaFluor 488-conjugated secondary antibodies (both 1:200). For co-staining, cell samples were stained with anti- $\gamma$-H2AX (1:400) and anti-p65 (1:400) antibodies followed by incubation with secondary antibodies (1:200). Cell nuclei were counterstained with Hoechst or DAPI. Fluorescence intensity was examined using a confocal scanning microscope (BX41F; Olympus, Tokyo, Japan).

ELISA. At the indicated time points post-irradiation, the culture medium was collected and the levels of IL-1 $\beta$, IL-6, TNF- $\alpha$ and TGF- $\beta$ were determined by ELISA.

RNA extraction and real-time reverse transcriptase polymerase chain reaction $(R T-P C R)$. Total RNA was extracted from cultured BV-2 cells using TRIzol reagent. The sequences of the specific primers used were designed by Beacon software (Bio-Rad Laboratories) and were as follows, with the forward primer followed by the reverse primer for each target: mouse TNF- $\alpha$ 5' primer, TTC TCA TTC CTG CTT GTG G and 3' primer, CTT GGT GGT TTG CTA CGA C; mouse IL-1 $\beta 5^{\prime}$ primer, AAA TCT CGC AGC AGC ACA T and 3' primer, CAC ACA CCA GCA GGT TAT CA; mouse IL-6 5' primer, TTG CCT TCT TGG GAC TGA T and 3' primer, TTG CCA TTG CAC AAC TCT T; mouse COX-2 5' primer, GAG TGG GGT GAT GAG CAA and 3' primer, GCA ATG CGG TTC TGA TAC T; GAPDH 5' primer, TCA CCA CCA TGG AGA AGG C and 3' primer, GCT AAG CAG TTG GTG GTG CA; 


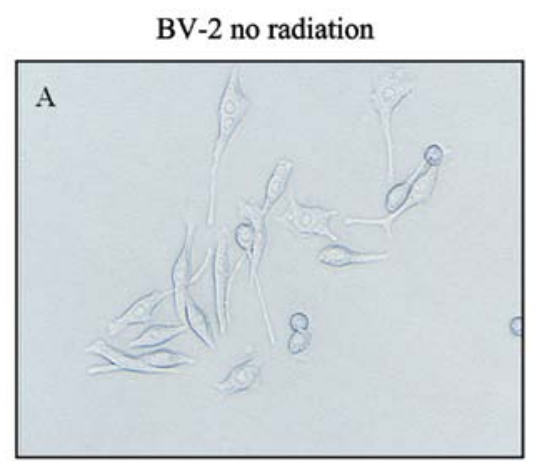

BV-2 + 16 Gy radiation

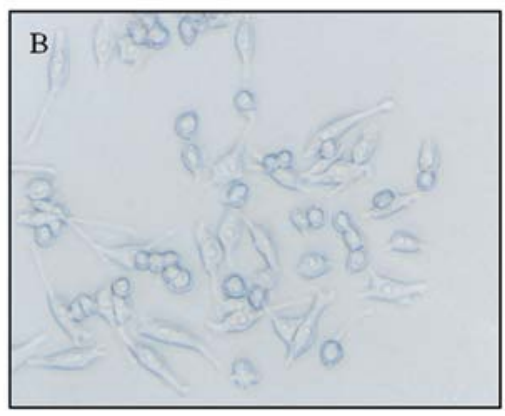

Figure 1. Morphology of control and irradiation-activated BV-2 cells. (A) Cells without radiation treatment. (B) Cells treated with 16 Gy radiation. Original magnification, $\mathrm{x} 400$.

mouse toll-like receptor (TLR)-8, 5' primer, GCC ACT GTG ACT AAT GGT CCT AA and 3' primer, CAC CAA CGC AAG CCA AAA TAA ATG. PCR amplification was performed using a Stratagene Mx3000P QPCR System (La Jolla, CA, USA). The thermocycling conditions were: 50 cycles at $94^{\circ} \mathrm{C}$ for $30 \mathrm{sec}, 57^{\circ} \mathrm{C}$ for $30 \mathrm{sec}$ and then $72^{\circ} \mathrm{C}$ for $30 \mathrm{sec}$. The relative expression from amplified RNA samples was calculated using the $2^{-\Delta \Delta \mathrm{CT}}$ method.

Immunoblotting. Nuclear or cytoplasmic protein was extracted from BV-2 cells using lysis buffer. Protein concentrations were measured using the bicinchoninic acid (BCA) assay. Equal amounts of protein lysates were diluted in sample loading buffer at a ratio of $4: 1$, heated to $95^{\circ} \mathrm{C}$ for $5 \mathrm{~min}$, separated by $12 \%$ sodium dodecyl sulfate-polyacrylamide gel electrophoresis (SDS-PAGE) and transferred to a nylon membrane. Membranes were blocked with $5 \% \mathrm{w} / \mathrm{v}$ non-fat dry milk in phosphate-buffered saline (PBS) plus Tween-20 (PBS-T) for $2 \mathrm{~h}$ and then incubated with an NF- $\mathrm{B}$ p 65 primary antibody $(1: 200)$ at $4^{\circ} \mathrm{C}$ overnight. After washing with PBS-T, membranes were incubated with HRP-conjugated secondary antibody for $1.5 \mathrm{~h}$ at room temperature. The results were detected using Kodak film (Rochester, NY, USA).

Statistical analysis. Data were analyzed with the Statistical Program for Social Sciences software (version 10.0; SPSS, Inc., Chicago, IL, USA) and were expressed as the mean \pm standard deviation (SD). Differences between groups were determined using the analysis of variance (ANOVA) or Student's t-test. $\mathrm{P}<0.05$ was considered to indicate a statistically significant difference.
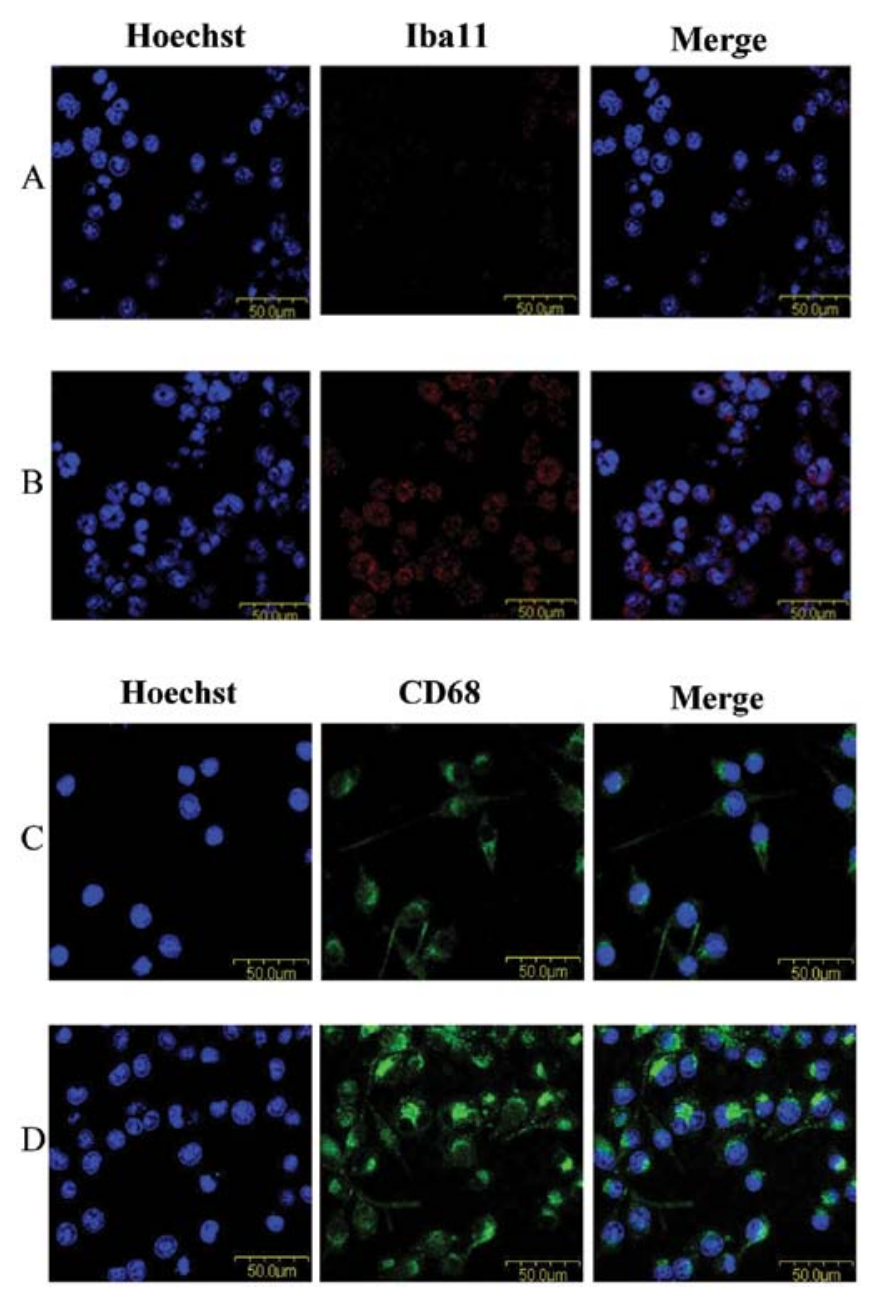

Figure 2. Expression of Iba-1 and CD68 in irradiated BV-2 cells. Control cells (upper panels) or irradiated-cells (lower panels) were immunostained with anti-Iba-1 antibody (A and B; red) or anti-CD68 (C and D; green). Cellular nuclei were stained with Hoechst (blue). Scale bars, $50 \mu \mathrm{m}$.

\section{Results}

Microglial activation induced by irradiation. Control BV-2 microglial cells had a small cell body and multiple long processes, showing a typical ramified structure (Fig. 1A). Low-dose radiation ( $<16 \mathrm{~Gy}$ ) had no effect on the microglial morphology of these cells (data not shown). However, $30 \mathrm{~min}$ following $16 \mathrm{~Gy}$ of radiation, the microglia appeared to be activated, with cells exhibiting a stouter spherical morphology (Fig. 1B). The levels of both Iba-1 and the activation marker CD68 were greatly upregulated in cells following irradiation (Fig. 2), indicating microglia activation can be induced by radiation treatment.

Radiation-induced alteration of inflammatory cytokinesis in microglia. We next examined the levels of inflammatory factors, including IL-1 $\beta$, TNF- $\alpha$, IL-6, TLR- 8 and COX-2, following irradiation. BV-2 microglial cells were irradiated with $32 \mathrm{~Gy}$. As revealed by the ELISA results in Fig. 3A and the real-time PCR results in Fig. 3B, the levels of IL- $1 \beta$, TNF- $\alpha$, IL-6, TLR-8 and COX-2 in control microglia were relatively low. The levels of these inflammatory factors increased 

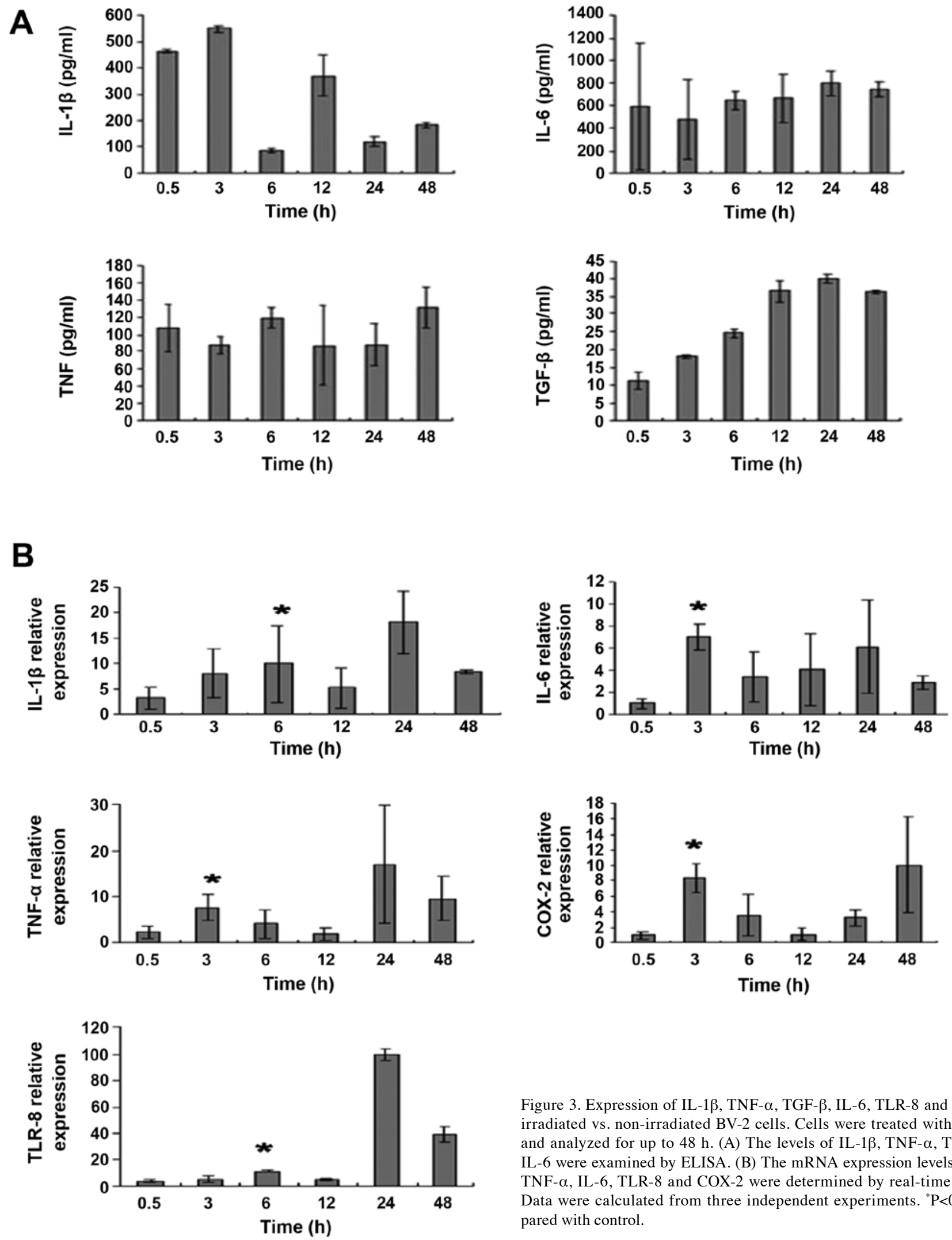

Figure 3. Expression of IL-1 $\beta$, TNF- $\alpha$, TGF- $\beta$, IL-6, TLR- 8 and COX-2 in irradiated vs. non-irradiated $\mathrm{BV}-2$ cells. Cells were treated with radiation and analyzed for up to $48 \mathrm{~h}$. (A) The levels of IL-1 $\beta$, TNF- $\alpha$, TGF- $\beta$ and IL-6 were examined by ELISA. (B) The mRNA expression levels of IL-1 $\beta$, TNF- $\alpha$, IL-6, TLR-8 and COX-2 were determined by real-time RT-PCR. Data were calculated from three independent experiments. " $\mathrm{P}<0.05 \mathrm{com}-$ pared with control.

post-irradiation, peaking at $3 \mathrm{~h}$ (IL-6, TNF- $\alpha$ and COX-2) or $6 \mathrm{~h}$ (IL-1 $\beta$ and TLR-8) $(\mathrm{P}<0.05$ compared with control). Upregulation of these factors was still detected at 24 or $48 \mathrm{~h}$ following irradiation.
Expression of $\gamma-H 2 A X$ and $N F-\kappa B$ p65 in irradiated $B V-2$ cells. Since activated $\gamma-\mathrm{H} 2 \mathrm{AX}$ that localizes to the nucleus facilitates DNA DSBs, we determined the expression of $\gamma$ - $\mathrm{H} 2 \mathrm{AX}$ as well as the activity of the transcription factor 
A
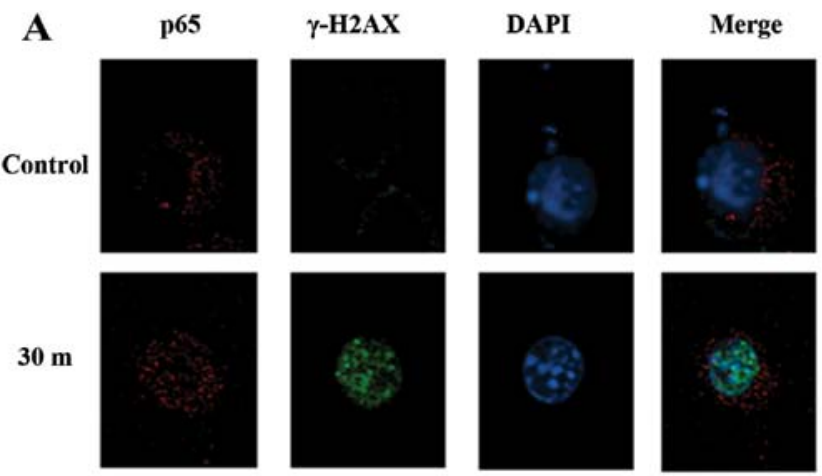

$3 \mathbf{h}$
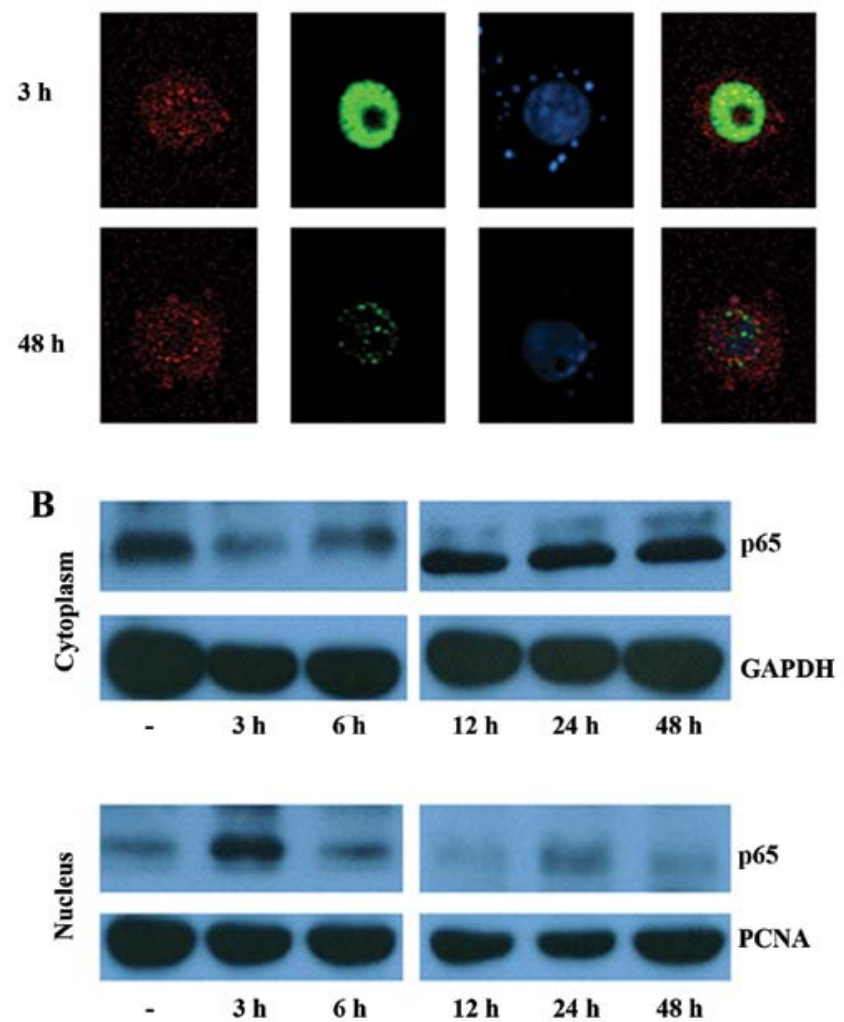

Figure 4. Expression of $\gamma-\mathrm{H} 2 \mathrm{AX}$ and $\mathrm{NF}-\kappa \mathrm{B}$ p65 in irradiated BV-2 cells. (A) Following $32 \mathrm{~Gy}$ irradiation for $0.5,3$ or $48 \mathrm{~h}$, cells were immunostained with anti- $\gamma$-H2AX (green) and anti-p65 (red) antibodies. Nuclei were counterstained with DAPI (blue). Original magnification, x1,000. (B) At the indicated time points following irradiation, the protein expression of p65 in the cytoplasm and nuclei was evaluated via immunoblot analysis.

$\mathrm{NF}-\kappa \mathrm{B}$. As shown in Fig. 4A, very little $\gamma-\mathrm{H} 2 \mathrm{AX}$ was detected in non-irradiated BV-2 cells. However, 30 min following $32 \mathrm{~Gy}$ of radiation treatment, marked upregulation of $\gamma$-H2AX was observed. In addition to this significant upregulation, $\gamma-\mathrm{H} 2 \mathrm{AX}$ was found to be localized to the nucleus, as can be seen from its co-localization with the nuclear DAPI stain (Fig. 4A). Enhanced $\gamma$-H2AX expression was detected $3 \mathrm{~h}$ post-irradiation but decreased after $48 \mathrm{~h}$. In control cells, the p65 subunit of NF- $\mathrm{BB}$ was primarily localized to the cytoplasm. Nuclear translocation of p65 was detected 30 min post-irradiation ( $32 \mathrm{~Gy}$ ) and peaked at $3 \mathrm{~h}$. However, $48 \mathrm{~h}$ after radiation treatment, nuclear translocation of p65 was reduced. Similar trends were observed in the cytoplasmic and nuclear expression of p65 as determined by immunoblot analysis (Fig. 4B).

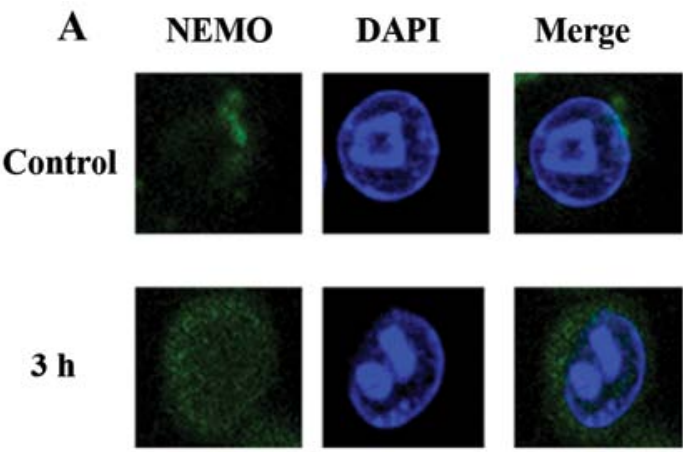

B
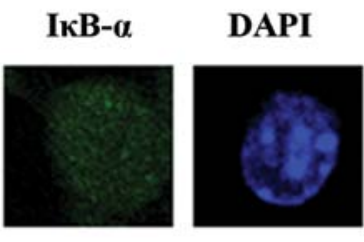

Merge
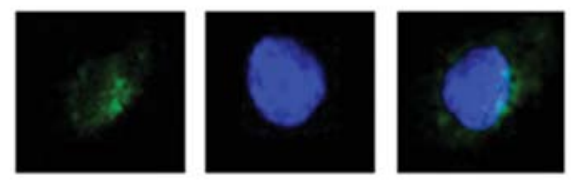

Figure 5. Expression of NEMO and I $\mathrm{B}-\alpha$ in irradiated BV-2 cells. Three hours following $32 \mathrm{~Gy}$ irradiation, cells were immunostained with anti-NEMO (A; green) and anti-IкB- $\alpha$ (B; green) antibodies. Nuclei were counterstained with DAPI (blue). Original magnification, x1,000. NEMO, $\mathrm{NF}-\kappa \mathrm{B}$ essential modulator.

Expression of NEMO and $I \kappa B-\alpha$ in irradiated $B V-2$ cells. $\mathrm{NEMO}$ and I $\kappa \mathrm{B}-\alpha$ are both important mediators of the $\mathrm{NF}-\kappa \mathrm{B}$ pathway. Immunocytochemical analysis indicated that NEMO fluorescence was diffusely distributed in the cytoplasm at a relatively low density under normal conditions (Fig. 5A). Three hours following 32 Gy irradiation, the NEMO protein was markedly elevated. Moreover, I $\kappa \mathrm{B}-\alpha$ levels were considerably decreased following irradiation (Fig. 5B). This evidence suggests that activation of NEMO and degradation of IкB- $\alpha$ occur after radiation treatment of microglial cells.

\section{Discussion}

Partial or whole brain irradiation is a widely used, effective treatment for primary and metastatic brain tumors. However, it may increase the risk of radiation-induced brain injury, including cognitive impairment with the manifestation of functional deficits in memory, attention and executive function that severely affect the patient's quality of life (14). Therefore, it is critical to both understand and minimize the side-effects of brain irradiation.

It has been reported that radiation may trigger activation of the microglia, which releases pro-inflammatory cytokines, subsequently inhibiting proliferation of neural precursor cells and hippocampal neurogenesis $(15,16)$. Here, we observed that irradiation at doses over 16 Gy could efficiently induce microglial activation and increase expression of the microglial biomarkers Iba-1 and CD68. In addition, the generation of pro-inflammatory factors, including IL-1 $\beta$, TNF- $\alpha$, IL-6, 
TLR-8 and COX-2, was greatly upregulated following irradiation. These results are consistent with previous reports that showed enhanced production of pro-inflammatory mediators within hours after brain irradiation $(6,11,17)$. Antiinflammatory agents, including ramipril and indomethacin, have been shown to decrease the number of activated microglia in the hippocampus and/or perirhinal cortex and prevent radiation-induced cognitive impairment in rodents $(15,18)$. Future studies will continue to explore the potential effects of anti-inflammatory drugs on brain injury induced by irradiation.

DNA DSBs, which are closely associated with phosphorylated $\gamma-\mathrm{H} 2 \mathrm{AX}$, represent a radiation-induced lesion (19). Ataxia telangiectasia mutated (ATM) is a nuclear protein kinase that mediates apoptosis and cell cycle checkpoint responses after DNA DSBs $(20,21)$. Moreover, it also represents a crucial regulator of NF- $\kappa B$ signaling pathway activation by mediating the activities of NEMO (which modulates NF- $\kappa B$ ) and I $\kappa B$ kinase

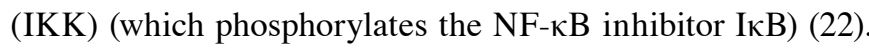
In the present study, we found that $3 \mathrm{~h}$ following irradiation, $\gamma$-H2AX was upregulated and the NF- $\mathrm{KB}$ p65 subunit had undergone translocation to the nucleus. Additionally, the $\mathrm{NF}-\kappa \mathrm{B}$ modulator NEMO was markedly elevated, whereas the $\mathrm{NF}-\kappa \mathrm{B}$ regulatory inhibitor I $\mathrm{KB}-\alpha$ was considerably decreased. Collectively, this indicates that the NF- $\kappa B$ signaling pathway is activated and may contribute to microglial activation upon radiation treatment. Activation of NF- $\mathrm{KB}$, specifically nuclear translocation of the p65 subunit, is required for the induction of several pro-inflammatory cytokines such as TNF- $\alpha$ (23). Antisense p65 oligonucleotides have been shown to reduce proinflammatory cytokine production (24). Based on these observations, we hypothesize that radiation increases NEMO activity, decreases the inhibitory effect of I $\mathrm{B}$, triggers the nuclear translocation of p65 and subsequently increases the upregulation of pro-inflammatory cytokines, such as IL-1 $\beta$ and TNF- $\alpha$.

In summary, we conclude that radiation at doses over $16 \mathrm{~Gy}$ can efficiently induce microglial activation through activating the NF- $\mathrm{\kappa B}$ signaling pathway and promoting inflammatory factor release. Future studies will focus on exploring the precise association between the NF- $\mathrm{kB}$ pathway and inflammation in activated microglia.

\section{Acknowledgements}

This study was supported by grants from National Natural Science Foundation of China (No. 30800283 and 81172595), Postdoctor Foundation of China (No. 20100480905) and Postdoctor Special Foundation of China (201104440).

\section{References}

1. Tofilon PJ and Fike JR: The radioresponse of the central nervous system: a dynamic process. Radiat Res 153: 357-370, 2000.
2. van Rossum D and Hanisch UK: Microglia. Metab Brain Dis 19: 393-411, 2004.

3. Greene-Schloesser D, Robbins ME, Peiffer AM, Shaw EG, Wheeler KT and Chan MD: Radiation-induced brain injury: a review. Front Oncol 2: 73, 2012.

4. Chiang CS, McBride WH and Withers HR: Radiation-induced astrocytic and microglial responses in mouse brain. Radiother Oncol 29: 60-68, 1993.

5. Hwang SY, Jung JS, Kim TH, et al: Ionizing radiation induces astrocyte gliosis through microglia activation. Neurobiol Dis 21: 457-467, 2006

6. Lee WH, Sonntag WE, Mitschelen M, Yan H and Lee YW: Irradiation induces regionally specific alterations in pro-inflammatory environments in rat brain. Int J Radiat Biol 86: 132-144, 2010.

7. Pocock JM and Liddle AC: Microglial signalling cascades in neurodegenerative disease. Prog Brain Res 132: 555-565, 2001.

8. Kim SU and de Vellis J: Microglia in health and disease. J Neurosci Res 81: 302-313, 2005.

9. Dropcho EJ: Neurotoxicity of radiation therapy. Neurol Clin 28: 217-234, 2010.

10. Kyrkanides S, Olschowka JA, Williams JP, Hansen JT and O'Banion MK: TNF- $\alpha$ and IL-1 $\beta$ mediate intercellular adhesion molecule-1 induction via microglia-astrocyte interaction in CNS radiation injury. J Neuroimmunol 95: 95-106, 1999.

11. Kyrkanides S, Moore AH, Olschowka JA, et al: Cyclooxygenase-2 modulates brain inflammation-related gene expression in central nervous system radiation injury. Brain Res Mol Brain Res 104: 159-169, 2002.

12. Heese K, Fiebich BL, Bauer J and Otten U: NF- $\mathrm{B}$ modulates lipopolysaccharide-induced microglial nerve growth factor expression. Glia 22: 401-407, 1998.

13. Liu B, Wang K, Gao HM, Mandavilli B, Wang JY and Hong JS: Molecular consequences of activated microglia in the brain: overactivation induces apoptosis. J Neurochem 77: 182-189, 2001.

14. Greene-Schloesser D, Moore E and Robbins ME: Molecular pathways: radiation-induced cognitive impairment. Clin Cancer Res 19: 2294-2300, 2013

15. Monje ML, Toda H and Palmer TD: Inflammatory blockade restores adult hippocampal neurogenesis. Science 302: 1760-1765, 2003.

16. Monje ML, Mizumatsu S, Fike JR and Palmer TD: Irradiation induces neural precursor-cell dysfunction. Nat Med 8: 955-962, 2002.

17. Chiang CS, Hong JH, Stalder A, Sun JR, Withers HR and McBride WH: Delayed molecular responses to brain irradiation. Int J Radiat Biol 72: 45-53, 1997.

18. Lee TC, Greene-Schloesser D, Payne V, et al: Chronic administration of the angiotensin-converting enzyme inhibitor, ramipril, prevents fractionated whole-brain irradiation-induced perirhinal cortex-dependent cognitive impairment. Radiat Res 178: 46-56, 2012.

19. Lobrich M, Shibata A, Beucher A, et al: $\gamma \mathrm{H} 2 \mathrm{AX}$ foci analysis for monitoring DNA double-strand break repair: strengths, limitations and optimization. Cell Cycle 9: 662-669, 2010.

20. Abraham RT: Cell cycle checkpoint signaling through the ATM and ATR kinases. Genes Dev 15: 2177-2196, 2001.

21. Bakkenist CJ and Kastan MB: Initiating cellular stress responses. Cell 118: 9-17, 2004.

22. Wu ZH, Shi Y, Tibbetts RS and Miyamoto S: Molecular linkage between the kinase ATM and NF- $\mathrm{BB}$ signaling in response to genotoxic stimuli. Science 311: 1141-1146, 2006.

23. Beg AA and Baltimore D: An essential role for NF- $\mathrm{BB}$ in preventing TNF- $\alpha$-induced cell death. Science 274: 782-784, 1996.

24. Tak PP and Firestein GS: NF-кB: a key role in inflammatory diseases. J Clin Invest 107: 7-11, 2001 